http://dx.doi.org/10.21611/qirt.1994.014

\title{
Discussion of different numerical models applied to air infiltration measurement in external walls.
}

by DUC E. *

\section{* Technical University of Wroclaw, Instifute of Building Engineering, Poland}

Abstract

When computing heat loss or temperature fields in external wall joints, with existing cracks, numerical mesh layout is chosen depending upon crack type. In practice, analytic approach can be used only in case of uniform walls or sandwich wall (with no head leakage bridge) with a straight crack coming through. The paper summarizes comparison of results developed according to numerical outcome of the balance analysis for different practical mesh layouts. The results can be applied to in situ research of external wall joint air tightness using thermal - pressure method [1], where thermovision equipment ( 880 LWB + TIC 8000) is used.

Nomenclature

c heat capacity

d thickness

$h$ network step

$h_{s}$ thickness of crack

$I_{z} \quad$ air permeability in joint

$v$ velocity of air

$t$ temperature,

\section{Greek symbols}

$\lambda$ thermal conductivity

$\rho$ density

$\tau$ time

$\alpha$ convective heat transfer

$\alpha_{p}$ coefficient of heat transfer in the crack

$\vartheta$ temperature in the node,

\section{Subscripts}

a air

i,j,n natural numbers

p permeability

s crack

$i \quad$ inside

e exterior

\section{Description of models}

\subsection{Analytical model}

Solution is obtained in form of non-dimensional temperature $\theta$ in the area of equivalent wall with thickness $d_{1}$, obtained from applying Fourier method to the Laplace equation [2]:

$$
\vartheta(x, y)=x+\sum_{n=1}^{\infty} C_{n} \exp (-n \pi y) \sin n \pi x,
$$

where:

$$
C_{n}=\frac{\frac{n \pi\left[1-(-1)^{n} \exp (-\beta)\right]}{\beta^{2}+n^{2} \pi^{2}}-\frac{1-(-1)^{n}}{n \pi}}{\beta\left\{\frac{1}{2}\left(1+\frac{n \pi \lambda h_{s}}{\mathrm{Nu} \lambda_{a} d_{1}}\right)-\frac{n^{2} \pi^{2} \beta\left[1-(-1)^{n} \exp (-\beta)\right]}{\beta^{2}+n^{2} \pi^{2}}-\frac{\beta^{2}}{2\left(\beta^{2}+n^{2} \pi^{2}\right)}\right\}}
$$

$\mathrm{C}_{n}$ values take into consideration the boundary conditions as a function of nondimensional Nusselt number $\mathrm{Nu}$ and Peclet number $\mathrm{Pe}\left(\mathrm{Pe}=v h_{s} c_{a} \rho_{a}\right)$ where $\beta=2 \mathrm{Nud} /$ $\left(P e h_{s}\right)$. Abscissa $x$, calculated proportionally to the wall on the internal surface in nondimensional coordinates, has a value of $(1-1 / \mathrm{Bi})$, where $\mathrm{Bi}=\alpha_{1} d_{1} / \lambda$. For the cases where $h_{s}$ $<0.002 \mathrm{~m}$, which corresponds to practical values of $\alpha_{s} \mathrm{Bi} / \alpha_{l}>35$, with the estimated error 
http://dx.doi.org/10.21611/qirt.1994.014

value not higher than $1 \%$ a simplified formula for non-dimensional temperature can be used: $\Delta \Theta=\left(\vartheta_{1}-\vartheta_{l, s}\right) /\left(\vartheta_{l}-t_{0}\right)$

$$
\Delta \Theta=\left[1-\left(\frac{\alpha_{s}}{\alpha_{i}} \mathbf{B i}\right)^{-1 / 3}\right]\left\{1-\exp \left[-\frac{1-\ln \frac{\pi}{2 \mathbf{B i}}}{\pi(\mathbf{B i}-1)\left[1-\left(\frac{\alpha_{s}}{\alpha_{i}} \mathbf{B i}\right)^{-1 / 3}\right]} \cdot \frac{\lambda_{a} \mathbf{P e}}{\lambda}\right]\right\}
$$

Equation (3) is more suitable for comparison because it takes into consideration the coefficient of heat transfer in the crack $\alpha_{s}$. Most frequently used value of $\mathrm{Nu}$, on which $\alpha_{s}$ coefficient depends, varies from 4.0 to 7.6.

\section{2. Numerical models}

As mentioned before, from the practical point of view balance equations are constructed for different mesh layouts, depending on shape of the cracks. For the purpose of further analysis three different kinds of mesh structures were used shown on fig. 1 as "a", "b", " $c$ " with balancing element marked. Model " $b$ " corresponds to that used in the literature so far, while models "a" and " $c$ " are introduced by the author. For the clarity purpose the " $b$ " model shall be described. Equations will be shown as for the transient state, to be used further on in the final conclusions.

The " $b$ " model has been used among others in [3], where the crack divides the evaluated region into two symmetrical parts with nodes situated along crack axis and on its surface. Temperature in the nodes of crack axis $t_{s}$ in $(n+1)$ time period $\Delta \tau$ for the infiltration is evaluated from the equation:

$2 \alpha_{p, n}\left(t_{s, i, j, n+1}-t_{s, i, j-1, n}\right) \Delta \tau / h=2 \alpha_{s, n}\left(\vartheta_{s, i-1, j, n}-t_{s, i, j, n+1}\right) \Delta \tau$

and used in balance equation for crack surface temperature $\vartheta_{\mathbf{s}}$ :

$$
\begin{aligned}
\vartheta_{s, i-1, j, n+1} & =\vartheta_{s, i-1, j, n}+\Delta \tau\left[\lambda\left(2 \vartheta_{i-2, j, n}+\vartheta_{i-1, j-1, n}+\vartheta_{i-1, j+1, n}-4 \vartheta_{i-1, j, n}\right)+\right. \\
& \left.+2 \alpha_{s} h\left(t_{s, i, j, n+1}-\vartheta_{s, i-1, j, n}\right)\right] /\left(c \rho h^{2}\right) .
\end{aligned}
$$

In model "a", axis temperature is a result of a balance equation with the assumption that in narrow cracks $\left(h_{s} \rightarrow 0\right)$, the temperature at crack surface $\vartheta_{s}$ approaches the temperature in the node off $\operatorname{crack}\left(\vartheta_{s} \rightarrow \vartheta_{i, j}\right)$, while infiltrating air temperature by node off crack $\left(t_{s, i J-1, n} \rightarrow \theta_{i J-1, n}\right)$, see models "a" and " $b$ ", fig. 1 . Following these assumptions, equation (4) takes the shape of:

$2 \alpha_{p, n}\left(t_{s, i, j, n+1}-\vartheta_{i, j-1, n}\right) \Delta \tau / h=2 \alpha_{s, n}\left(\vartheta_{i, j, n}-t_{s, i, j, n+l}\right) \Delta \tau$, 


$$
\begin{aligned}
\vartheta_{i, j, n+1} & =\vartheta_{i, j, n}+\Delta \tau\left[\lambda\left(\vartheta_{i-1, j, n}+\vartheta_{i+1, j, n}+\vartheta_{i_{2},-1, n}+\vartheta_{i, j+1, n}-4 \vartheta_{i, j, n}\right)+\right. \\
& \left.+2 \alpha_{s} h\left(t_{s, i, j, n+1}-\vartheta_{i, j, n}\right)\right] /\left(c \rho h^{2}\right) .
\end{aligned}
$$

In model " $c$ ", $(i-1)$ node row is situated at distance from crack surface, which leads to head resistance increase between crack axis nodes and the first row of nodes parallel to crack axis. Crack axis temperature, which at the same time equals temperature in the $(i, j)$ node retaining the balance, is shown in [1]:

$$
\begin{aligned}
\vartheta_{i, j, n+1} & =\frac{\Delta \tau}{h^{2} c \rho}\left\{(\lambda+A) \vartheta_{i, j-1, n}+\left[\frac{h^{2} c \rho}{4 \Delta \tau}+(\lambda-B)\right] \vartheta_{i, j, n}+(\lambda-C) \vartheta_{i, j+1, n}+\right. \\
& +\left[\frac{h^{2} c \rho}{4 \Delta \tau}-(\lambda+D)\right] \vartheta_{i, j, n}+\frac{\lambda \alpha_{s} h}{\left(\alpha_{s} h+\lambda\right)} \vartheta_{i-1, j, n}+ \\
& \left.+\left[\frac{h^{2} c \rho}{2 \Delta \tau}-\frac{2 \lambda \alpha_{s} h}{\left(\alpha_{s} h+\lambda\right)}\right] \vartheta_{i, j, n}+\frac{\lambda \alpha_{s} h}{\left(\alpha_{s} h+\lambda\right)} \vartheta_{i+1, j, n}\right\}
\end{aligned}
$$

Assuming that infiltrating air temperature by the element under analysis equals that of backward node $(i, j-1)$ and exfiltrating air temperature equals that of the central node $(i, j): A=$ $2 \alpha_{p}, B=-A, C=D=0$, where $\alpha_{p}$ similarly to earlier models equals half the product value of air volume permeability of the crack $I_{z}$ times specific heat $c_{a}$ and air density $\rho_{a}\left(\alpha_{p}=I_{z} c_{a} \rho_{a} / 2\right)$.

In case of model " $c$ " heat transmission in the direction towards crack axis, e. $g$. between nodes $(i-1, j)$ and $(i, j)$ covers also heat flow resistance $1 / \alpha_{s}$, which is accounted for in balance equations for nodes of $(i-1)$ row.

\section{Model evaluation by test tasks}

While testing the correctness of numerical models used (" $a$ ", " $b$ " and "c"), evaluations were carried out regarding infiltration of crack through external, uniform wall $\left(h_{s}=0.001 \mathrm{~m}, \mathrm{Nu}\right.$ $=7.6)$. Three different step values of the mesh were used $(h=0.009 ; 0.0045$ and $0.003 m)$, as well as two values of heat transfer coefficient within the crack $\alpha_{p}=0.01(\mathrm{Pe}=0.8189)$ and $\alpha_{p}=0.02(\mathrm{Pe}=1.6378)$. Models were examined as for the transient state with constant boundary conditions, which allows for comparison of results with the simplified analytic formula (3). Analyzing the phenomenon at constant temperature values on both sides of the uniform wall the researchers wanted also to measure time needed before two temperature fields become stable, with the assumption that for $\tau=0, \alpha_{p}=0$ and for $\tau>0, \alpha_{p}=$ const.

As starting condition, it has been assumed that in the whole region, for $\tau=0$, the distribution of temperature is stable and results from unidirectional heat flow in the wall (with no account for the crack). For $\tau>0$ temperature at the crack inlet, as well as heat transfer coefficient within the crack, are constant and amount to $t_{\theta}$ and $\alpha_{p}$ respectively. The remaining data values show as follows: $\lambda=0.042 \mathrm{~W} /(\mathrm{m} \mathrm{K}), d=0.054 \mathrm{~m}\left(d_{1}=0,061 \mathrm{~m}\right), t_{1}=20, t_{\theta}=-20$ ${ }^{\circ} \mathrm{C}, \alpha_{1}=8, \alpha_{s}=23, \alpha_{s}=92.8 \mathrm{~W} /\left(m^{2} K\right)$. In the regions off the crack standard balance equations applied. Evaluated step after time in accordance with [1] was assumed at $\Delta \tau=1 \mathrm{~s}$, except for model " $b$ ", with mesh $h=0.0045$ and $0.003 \mathrm{~m}$, where $\Delta \tau$ is established using trial method at $\Delta \tau$ $=0.5 \mathrm{~s}$. 
Computed results of $9_{i, s}$ during the whole of the period under study are shown on fig. 1 as non - dimensional temperature $\Delta \Theta$ for models "a", "b" and " $c$ ". Because of the fact that results for the meshes 0.003 and 0.0045 were similar, the latter are not included in the drawing. The value of $\Delta \Theta$ for $P e=0.8189$ computed from (3) amounts to 0.0415 , while numerical computations (for $h=0.003 \mathrm{~m}$ ) amount to: for model "a" $\rightarrow \Delta \Theta=0.0396$, for " $b$ " $\rightarrow$ $\Delta \Theta=0.0438$, for " $\mathrm{C}$ " $\rightarrow \Delta \Theta=0.0509$, which means that $\vartheta_{l, s}=15.11 ; 14.96 ; 14.70{ }^{\circ} \mathrm{C}$ accordingly, with analytically obtained value of $\vartheta_{l, s}=15.04{ }^{\circ} \mathrm{C}$. Similarly, analytically obtained value of $\Delta \Theta$ for $\mathrm{Pe}=1.6378$ is 0.0813 , while numerically model "a" $\rightarrow \Delta \Theta=0.0830$, "b" $\rightarrow \Delta \Theta$ $=0.0954$ and $" \mathrm{C} " \rightarrow \Delta \Theta=0.1105$, which, as above, results in $\vartheta_{l, s}=13.53 ; 13.08$ and $12.52{ }^{\circ} \mathrm{C}$ with analytically obtained $\vartheta_{l, s}=13.59^{\circ} \mathrm{C}$. While assigning values for $\Delta \Theta$ for models "a" and "C" instead of $\vartheta_{l, s}$ researchers used $\vartheta_{l, \downarrow, n}+1$ (along the crack axis by warm surface of interior face of the wall).

\section{Conclusions}

Narrow distribution of results allows for accepting all three models "a", "b", "c" for engineering calculations, with stress put on the first two ("a" and " $b$ ") being the most reliable in evaluating $\vartheta_{l, s}$ temperature. Model " $c$ " results in underrated values due to largest distance from the first node row from the crack axis. It may be treated as advantageous error (safety margin) when comparing e. g. $\vartheta_{l, s}$ temperature with dew - point value.

In the final stage of computation for $\tau=1200 \mathrm{~s}$, in all three models, increments of $\vartheta_{\text {, s }}$ temperature were $0.0001^{\circ} \mathrm{C}$ at time increment of $100 \Delta \tau$. This will result in comparatively fast stabilization of temperature field when wall with a crack is used, at constant temperature difference of $t_{i}$ and $t_{\theta}$, provided that air flow with constant flow rate through the crack is initiated.

\section{REFERENCES}

[1] DUC (E.), and JAWORSKI (J.). - Air in and exfiltration thought the joints of external Walls. Quantitative Infrared thermography QIRT 92, ONERA, Paris 1992, pp. 315-318

[2] SINDOROV (E:A.). - Thermal regime of non air-tight joints of prefabrication walls. CNTIGSA, Moscow, 10, 1981, pp. 1-19 (in Russian)

[3] TITOV (V.P.). - Thermal regime of non air-tight joints. Inz. - Fiz. Zurnal 8, 1965, pp. 156-160 (in Russian)

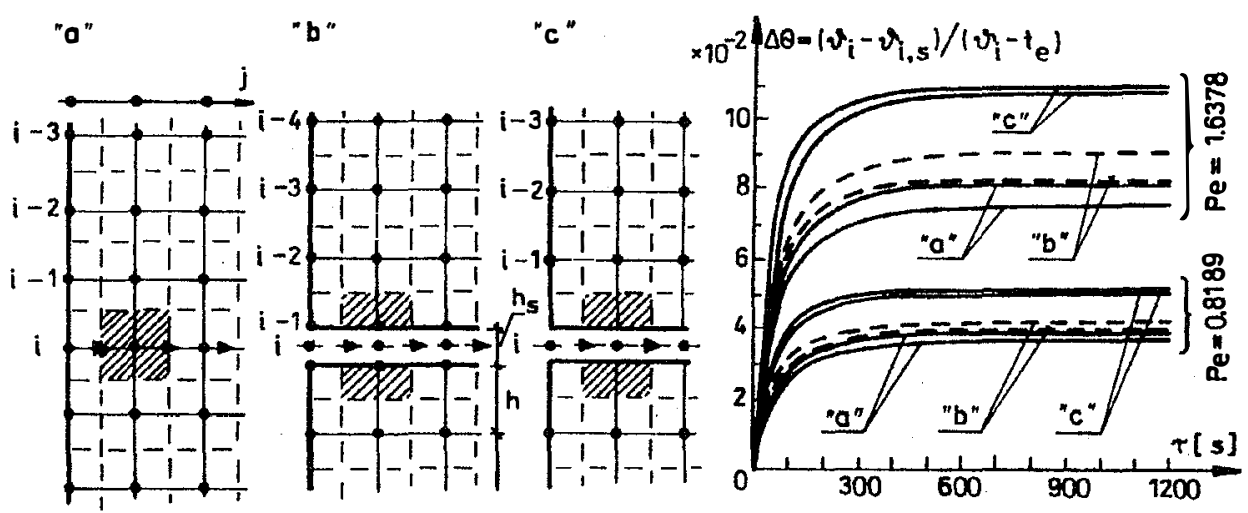

Fig. 1 Comparison of numerical calculation results for models "a", "b" and "c" at mesh step $h=0.009 m$ (upper curves) and $h=0.003 m$ (lower curves). 